Chapter 7

\title{
The Small Molecule Inhibitor of Protein Kinase Revolution for the Treatment of Rheumatoid Arthritis
}

\author{
Charles J. Malemud \\ Additional information is available at the end of the chapter \\ http://dx.doi.org/10.5772/59639
}

\section{Introduction}

Targeted medical intervention for the treatment of rheumatoid arthritis (RA) has significantly revolutionized clinical outcomes for this autoimmune disease. Until the development of biological drugs which have the capacity to block the activity of most of the critical proinflammatory cytokines involved in RA as well as a host of immune cell-mediated events, the medical therapy of RA was limited to the use of first-line treatments including, non-steroidal anti-inflammatory drugs, sulphasalazine, and several immunosuppressive agents, such as glucocorticoids, prednisone and dexamethasone, methotrexate, and anti-malarial drugs (e.g. hydroxychloroquine) [1-5]. Agents that blocked the proliferation of T-lymphocytes, such as leflunomide, abatacept and/or B-lymphocytes (e.g. rituximab) were also employed, but mainly as second-line therapies [6-9].

The momentous development of additional biological drugs for RA arose through the identification of those pro-inflammatory cytokines that were intimately involved in initiating and perpetuating the RA process. Thus, blocking tumor necrosis factor- $\alpha /$ tumor necrosis factor- $\alpha$ receptor, interleukin-1 $\beta$ (IL-1 $\beta$ ) IL-1 $\beta$ receptor and IL-6/IL-6 Receptor/gp130 signaling pathways with either monoclonal antibodies or engineered fusion proteins prominently entered into the armamentarium for the medical therapy of RA [10-15].

However, what does all of this mean for the future development of additional novel therapies for this chronic and debilitating synovial joint disease? For one thing the revolution in new drug development implies that although additional cellular targets including vascular endothelial growth factor (VEGF), adhesion molecules (e.g. vascular cell adhesion molecule-1; VCAM-1; CD106) and chemokines (e.g. CXC, C, CX3C and their corresponding receptors, CXCR, CCCR, CR and CX3CR) [16-26] which fit securely into a system of well-orchestrated RA pathophysiologic processes in man have been validated 
through in vitro studies as well as from impressive clinical results and ex vivo cell analyses with rodent models of human RA. Although none of the CC chemokine receptor antagonists have thus far yielded drugs effective in a rheumatology clinical practice, there are CXCR4 small drug antagonists in clinical trials for cancer, human immunodeficiency virus and the rare WHIM immunodeficiency condition [27].

One aspect in the study of human RA progression revolves around successfully translating from "hypothesis" to experimental validation and then going forward to drug development. This advancement has occurred by recognizing the crucial role played by signal transduction pathways in perpetuating the inflammatory state of RA. Thus, signal transduction has also been identified as the crucial cellular pathway that can cause "apoptosis-resistance" resulting in the aberrant survival of activated T-lymphocytes, B-lymphocytes and synoviocytes in RA synovial tissue [28, 29]. Signal transduction has also been identified as playing a role in the elevated frequency of apoptotic chondrocytes in RA articular cartilage [30, 31]. In that regard, blocking the phosphorylation of specific protein kinases involved signal transduction was explored as a mechanism to not only restore the appropriate balance between cell survival and cell death, which is skewed towards cell survival in RA, but also for blocking those protein kinases involved in up-regulating pro-inflammatory cytokine and matrix metalloproteinase (MMP) gene expression that is so integral to perpetuating inflammation and therefore, the destruction of synovial joints in RA [32-36].

Over the past 8 years or so, we have extensively detailed the genesis of interest in, and the extent to which, kinase activity of mitogen-activated protein kinases (MAPK) and the phosphatidylinositide-3-kinase/AKT/mammalian target of rapamycin (mTOR) (PI3K/AKT/mTOR) pathways influence MMP gene expression and cell survival, respectively [37-41]. We and others have also focused attention on the Janus Kinase/Signal Transducers and Activators of Transcription (JAK/STAT) pathway which was identified and targeted for drug development in RA because JAK/STAT signaling was found to play a major role in RA by promoting proinflammatory cytokine gene expression and abnormal cell survival [42-48]. Thus, this latter research focus resulted in the first JAK3-selective small molecule inhibitor (SMI), tofactinib, for use in the treatment of RA [reviewed in 49, 50].

This chapter will critically analyze the current state of drug development for novel protein kinase SMIs for RA, including a brief update and perspective on newer JAK SMI, besides tofacitinib, which are likely to be the targets for future drug development as well as additional protein kinase targets for RA.

\section{Newer JAK SMIs}

There are 4 members of the JAK family: JAK1, JAK2, JAK3 and Tyk2 [51]. JAK1 and JAK3 bind constitutively to the cytoplasmic region of the common gamma chain of cytokine receptors. This domain is the common subunit for many cytokines involved in T-cell and natural killer cell development in addition to B-cell activation making JAK1/JAK3 pertinent targets for intervention in RA [47, 52]. In addition to the JAK3-selective SMI, tofacitinib [54], several other 
JAK SMIs, with activity towards JAK 1 and JAK2, including baricitinib [55], CEP-33779 [55], INCB028050, PF-956980 [56, 57], filgotinib (INCB-039110), decernotinib, ruxolitinib, pefcitinib, ABT-494, INCB 047986 and AC-410 [58] are currently in various stages of preclinical testing and/or clinical assessment for altering the clinical course of RA, organ transplant rejection and psoriasis [59].

It has been widely held that JAK1 and JAK3 are essentially regulated by specific tyrosine sites within their respective activation loops [60], including the $\mathrm{NH}_{2}$-terminal kinase domain [45]. As such the activity of both JAK1 and JAK3 need be blocked to ensure inhibition of IL-2induced STAT5 activation [47]. This assertion is bolstered by evidence that JAK1 has a dominant role over JAK3 [61] likely making selective ATP-competitive JAK3 inhibitors less effective by themselves at the cellular level. Thus, the targeting of suppressor of cytokine signaling as a means to suppress JAK/STAT signaling $[45,62,63]$ is also being investigated for its potential to block cytokine-mediated STAT activation.

\section{Update on tofacitinib - 2014}

The approval of tofacitinib by the United States Food \& Drug Administration in 2012 for the treatment of moderate-severe RA has paved the way for the development of other JAK inhibitor compounds [64, 65]. The clinical data emerging from 4 Phase II and 4 Phase III RA clinical trials involving tofacitinib was recently summarized [Malemud CJ: Submitted]. In the Phase III RA trials where tofacitinib was compared to placebo a significant American College of Rheumatology-20 (ACR20) response was consistently demonstrated in the tofacitinib arm together with an improvement in the Health Assessment Questionnaire Disability Index and ACR50 response after 3 months of treatment with tofacitinib [66]. Most critically, these Phase III trials demonstrated that the clinical efficacy of tofacitinib was similar to the clinical responses achieved with the TNF inhibitor, adalimumab. Several common adverse events observed in these clinical trials were associated with the tofacitinib group and raised some concerns. This included an increase in the incidence of infections, infestations and creatinine along with an increase in the LDL-cholesterol/HDL-cholesterol ratio and decreased neutrophil counts. However, a recent meta-analysis of various RA clinical trials involving tofacitinib concluded that the frequency of adverse events was not increased by tofacitinib [67].

In another clinical trial where the clinical efficacy of tofacitinib was compared to methotrexate [68], 3 cases of confirmed lymphoma in 5 patients receiving tofacitinib compared to 1 subject in the methotrexate arm was reported. However, the ACR70 response was greater in the tofacitinib group compared to methotrexate, but the mean change in number of bone erosions in both the tofacitinib and methotrexate groups was modest. Importantly the subjects receiving tofacitinib had less cartilage loss at 6, 12 and 24 months of treatment.

Malemud and Blumenthal [50] recently reviewed the variety of cellular mechanisms that have been shown to be altered by tofacitinib which likely contributed to the clinical efficacy of the drug in human RA clinical trials, including the relative selectivity of tofacitinib for JAK1 and JAK3 over JAK2 in ameliorating the severity of arthritis in the rat-adjuvant model [69] as well 
as data showing that multiple cytokines and signaling pathways in addition to JAK/STAT were inhibited by tofacitinib at effective clinical doses which was distinct from conventional diseasemodifying antirheumatic drugs [70].

Although it is expected that the efficacy of tofacitinib in rheumatology clinical practice will have to be continuously monitored, tofacitinib will likely emerge as an important option for RA patients who inadequately respond to low-dose glucocorticoids, methotrexate and the several biologic drugs that are now routinely prescribed for the management of moderate-tosevere RA [50, 71, 72].

\section{Selective JAK inhibitors in development}

\subsection{Baricitinib}

Baricitinib (alternatively known as 1187594-09-7; INCB028050; UNII-ISP444213Y; LY3009104; LY-3009104 (Figure 1) [72] is a novel orally administered JAK inhibitor compound with relative selectivity for JAK1 and JAK2 [74]. In a study conducted among normal volunteers, baricitinib showed a dose-linear and time variant pharmacokinetics with low oral-dose clearance (i.e. 17L/h) and minimal systematic accumulation. Baricitinib also inhibited STAT3 phosphorylation in whole blood ex vivo, the results of which correlated with plasma concentrations of the drug. Although baricitinib was well-tolerated with negligible adverse events, the expected reduction in neutrophil counts was also recorded [75].

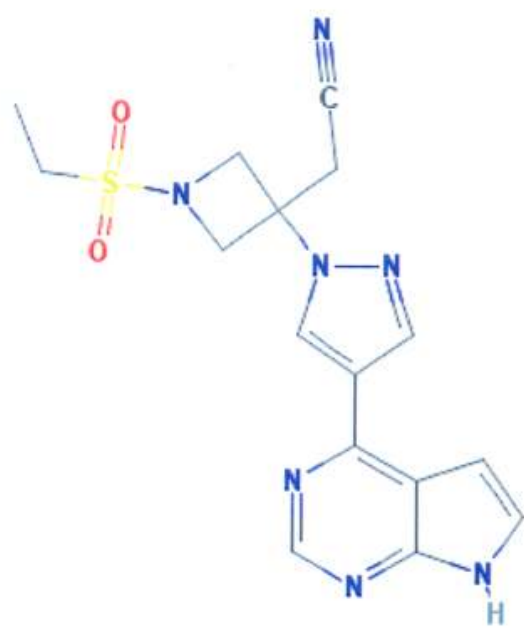

Figure 1. The chemical structure (IUPAC Name) of 2-[1-ethylsulfonyl-3-[4-(7H-pyrrolo[2,3-d]pyrimidin-4-yl)pyrazol-1yl]azetidin-3-yl]acetonitrile (Baricitinib) [73] 


\subsection{Decernotinib}

Decernotinib (Figure 2) alternatively known as VX-509, is an orally administered selective JAK3 inhibitor compound which is being evaluated for the possible medical management of RA $[58,76]$. In a recently completed study the results of which were reported at the 2014 European League Against Rheumatism (EULAR) annual meeting, the pharmacologic activity of decernotinib was compared to tofacitinib, filgotinib (GLPG 0634) and baricitinib with reference to the blockade of several cytokine signaling pathways in whole blood cell cultures from normal subjects. The cytokine pathways evaluated were the type I and type II interferon (i.e. INF $\alpha$ and INF $\gamma$ ) pathways, the common $\gamma$ chain cytokine pathways involving IL-15 and IL-21, and the IL-6 and IL-27-mediated signaling [77]. Although the results of this study showed that each of the JAK inhibitor compounds were relatively similar to each other in terms of their ability to block INF $\alpha$ and INF $\gamma$, IL-15, IL-21, IL-6 and IL-27 signaling, tofacitinib and baricitinib were more potent than decernotinib and filgotinib. Of note, these JAK inhibitor compounds exhibited lesser activity towards IL-10, IL-12, IL-23 and erythropoietin, which also signal via JAK/STAT [45]. Moreover, the results of the ex vivo studies evaluating the effect of these JAK inhibitor compounds on inhibition of cytokine signaling showed that they had markedly similar activities at clinically relevant doses.

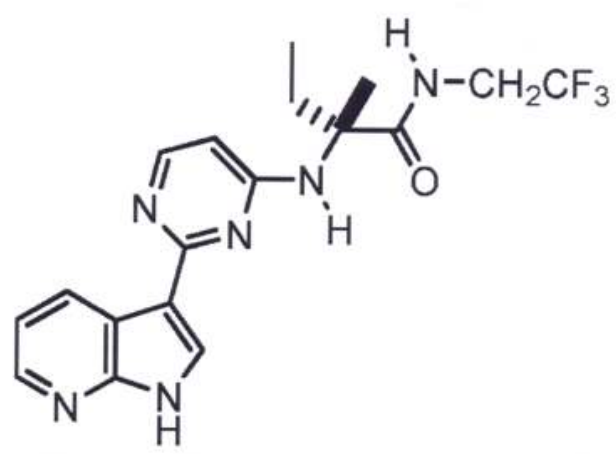

Figure 2. The chemical structure (IUPAC Name) of disodium N2-[2-(1H-Pyrrolo[2,3-pyridin-3-yl)pyrimidin-4-yl]-N(2,2,2-trifluoroethyl)-D-isovalinamide (Decernotinib) [76]

A recently completed 24-week randomized, placebo-controlled, double-blind, phase II study compared 4 dosing regimens of orally administered decernotinib given to 358 active RA patients, measured by increased C-reactive protein levels and in RA patients with at least 6 swollen and 6 tender joints who had an inadequate clinical response to methotrexate [78]. After 24 weeks, the clinical response to decernotinib was statistically significant by ACR20, ACR50 and ACR70 criteria and by positive changes in the Disease Activity Score-28 outcomes measure. However, the percentage of patients with any adverse event was higher in the decernotinib arm relative to placebo (i.e. a stable dose of methotrexate) which led to $9.1 \%$ of patients in the decernotinib group and 8.5 patients in the placebo group, respectively, to 
discontinue treatment. Of note, safety profiles were comparable across all dosage levels of decernotinib.

\section{Compounds that inhibit TyK2}

Tyrosine kinase-2 (TyK2) is the fourth member of the Janus kinase family. However, the development of TyK2-selective SMIs has lagged significantly behind the development of SMIs with activity against the other 3 members of the JAK family $[79,80]$. Thus, this remains an ongoing controversy for developing additional small compounds for RA despite the purported critical importance of TyK2 in driving IL-12-related cytokine IL-23 signaling which plays a key role in RA [81, 82].

In RA IL-23 is widely recognized as a key cytokine because of its role in perpetuating the inflammatory response as well as its involvement in the development of the IL-17-producing $\mathrm{T}_{\mathrm{H}} 17 \mathrm{~T}$-cell subset, the latter developing as a distinct $\mathrm{T}$-cell lineage apart from the cytokine-producing $\mathrm{T}_{\mathrm{H}} 1$ and $\mathrm{T}_{\mathrm{H}} 2$ T-cells [82]. Although the IL-23 receptor was the initial potential target for intervention in RA, inflammatory bowel disease, psoriasis and multiple sclerosis [83], a few laboratories focused on designing and synthesizing diamino 1, 2, 4 triazole compounds which could potentially demonstrate differential inhibition of TyK2 and JAKs 1-3 [81] or small molecule compounds such as APY0201 which was reported to directly inhibit IL-23 production [84].

Until recently, there were no patents filed for the design or production of selective TyK2 inhibitors. In that regard, both tofacitinib and one other small molecule compound, CMP6, failed to markedly inhibit TyK2. However, results with another small molecule compound, Cmpd1, showed some promise in preclinical analyses [85, 86]. For example, Qi et al. [87] recently demonstrated in human cervical cancer HeLa cells that apoptosis was enhanced after incubation of these cells with TNF- $\alpha$ but only if TNF- $\alpha$-induced heat shock protein (HSP)27 phosphorylation was suppressed by Cmpd1, or by MAPKAPK2 knockdown or by overexpression of a non-phosphorylatable HSP27 mutant, HSP27-3A. Thus, these results reported for Cmpd1 could indicate a role for TyK2 inhibition as influencing apoptosis in HeLa which may be useful to "cure" the apoptosis-resistance characteristic of RA synovial tissue [88]. However, in that study, HSP27 phosphorylation also facilitated TNF- $\alpha$ ubiquitination and phosphorylation of TAK1 as well as activation of p38 MAPK and ERK, the TAK1 pro-survival pathway downstream of p38 MAPK and ERK. Therefore, the extent to which further development of TyK2 small molecule inhibitor compounds such as Cmpd1 go forward will likely depend on its capacity to alter arthritis severity in rodent animal models of RA which will be solely dependent on the design and successful synthesis of selective TyK2 SMIs.

\section{The spleen tyrosine Kinase (SyK) inhibitor, fostamatinib}

SyK and the $\zeta$-chain associated protein-70 (ZAP-70) are non-receptor kinases which are preferentially produced by hemopoietic cells of the spleen, mast cells, polymorphonuclear 
leukocytes and macrophages [89]. In the context of adaptive immunity relevant to RA, Syk and ZAP-70 are major components in T-cell and B-cell receptor signaling [90]. Based on those findings, Syk was considered to be a promising target for RA primarily because of its involvement in regulating, not only T-cell and B-cell proliferation as well as those proliferating cells with the $\mathrm{F} \gamma$-activating receptor, but also in mediating immunoreceptor signaling by inflammatory cells and in signaling pathways regulated by immune complexes [91, 92].

Several years ago, Pine et al [93] showed that R788, which is the prodrug of the novel SyK SMI, R406, suppressed the severity of arthritis in collagen-induced arthritis (CIA), a well-validated mouse model of human RA. In addition to the finding that R788 showed significant clinical efficacy in mouse CIA, several surrogate molecules known to play important roles in inflammatory arthritis, namely the CXCR2 ligand KC-GRO- $\alpha$, macrophage chemoattractant protein-1, IL-1 and IL-6 were also reduced in mice with CIA treated with R788 compared to the vehicle control. Of note, the release of cartilage oligomeric matrix protein (COMP), a biomarker of extracellular matrix degradation in articular cartilage, was also suppressed by R788 suggesting a possible chondroprotective effect of R788.

More recent studies have been conducted to assess the pharmacokinetic/pharmacodynamic (PKPD) of R788, alternatively known as Fostamatinib disodium; R935788; R 935788 sodium; FosD, tamatinib fosdium (Figure 3) [94]. Results of these studies revealed that the converted form of the drug, R406, exhibited a PKPD relationship with changes in blood pressure [95]. Thus, the PKPD analysis revealed a concentration-dependent increase in blood pressure with increasing concentrations of R406. Nevertheless Baloum et al. [96] showed that R406 was rapidly absorbed with a terminal $t_{1 / 2}$ of 12-21 hrs. Furthermore, the solid dosage forms of fostamatinib provided a drug administration regimen whereby fostamatinib could be administered once daily or twice daily to achieve therapeutic levels of the drug.

What additional parameters of adaptive immunity were found to be altered by fostamatinib? Although R406 reduced the responsiveness of dendritic cells to immune complexes administered to mice, R406 did not reduce specific CD4 ${ }^{+} \mathrm{T}$-cell proliferation in these mice after immunization with these immune complexes [97]. However, R406 did reduce the interactions that occur between dendritic cells and antigen-specific CD4 ${ }^{+} \mathrm{T}$-cells. This resulted in reduced proliferation of these antigen-specific $\mathrm{CD} 4^{+} \mathrm{T}$-cells compared mice treated with the vehicle control. This change in antigen-specific $\mathrm{CD} 4^{+} \mathrm{T}$-cell proliferation in response to $\mathrm{R} 406$ was also characterized by a reduction in the level of several T-cell co-stimulatory biomarkers, namely, inducible T-cell co-stimulator and PD-1 as well as diminished production of the pro-inflammatory cytokines, INF- $\gamma$ and IL-17. Taken together, these $e x$ vivo results provided additional support for the indication that inhibiting SyK activity with fostamatinib could be an effective drug therapy to eliminate FcR-driven $\mathrm{CD} 4^{+} \mathrm{T}$-cells responses in RA.

So how did fostamatinib fare in RA clinical trials? The results of several RA clinical trials assessed the clinical efficacy of fostamatinib [98, 99]. Thus, Taylor et al. [98] reported that treatment of moderate-severe RA subjects with fostamatinib improved the DAS-28/C-reactive protein (CRP) score from baseline versus placebo injection at $6 \mathrm{wks}$ at 2 dosage regimens: 100 $\mathrm{mg}$ twice daily for $24 \mathrm{wks}$ plus placebo injection every $2 \mathrm{wks}$ and $100 \mathrm{mg}$ twice daily for $4 \mathrm{wks}$, followed by $150 \mathrm{mg}$ once daily up to wk 24. However, DAS-28/CRP failed to improve when 


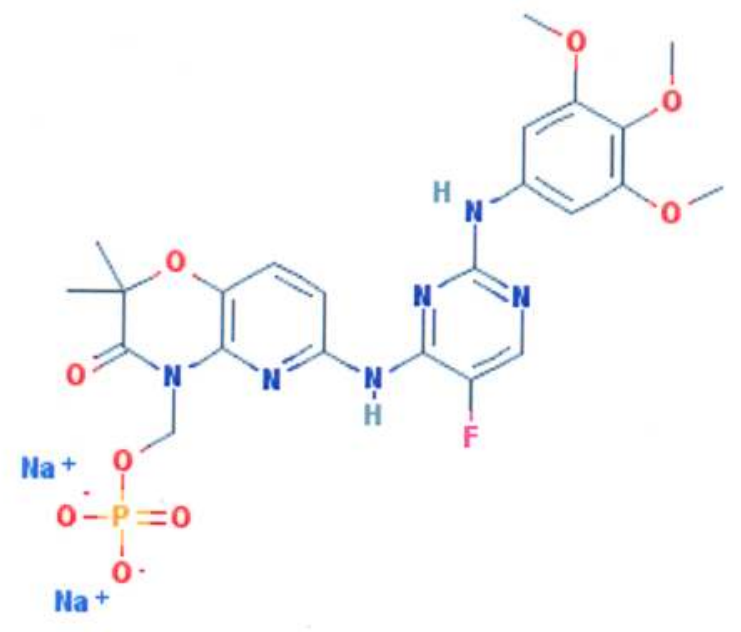

Figure 3. The chemical structure (IUPAC Name) of [6-[[5-fluoro-2-(3,4,5-trimethoxyanilino)pyrimidin-4-yl]amino]-2,2dimethyl-3-oxopyrido[3,2-b][1,4] oxazin-4-yl]methyl phosphate (Fostamatinib Sodium) [94]

fostamatinib was administered at $100 \mathrm{mg}$ twice daily for $4 \mathrm{wks}$, then $100 \mathrm{mg}$ once daily up to wk 24. Most critically, in a comparator analysis, fostamatinib was less effective than the TNF$\alpha$ blocker, adalimumab, at wk 24 based on the DAS-28/CRP criteria. The most common adverse effects of fostamatinib in this clinical trial were similar to those previously reported which included increased hypertension and diarrhea. Thus, although fostamatinib demonstrated significant clinical efficacy when employed as a monotherapy using DAS-28/CRP criteria at 6 wks, fostamatinib was inferior to adalimumab at wk 24 .

Three additional RA clinical trials measured the effectiveness of fostamatinib on subchondral bone erosions using the modified total Sharp score as an indicator of whether inhibition of SyK retarded the destruction of subchondral bone over a period of 6 months. ACR response criteria were also measured [99]. Once again, hypertension was the most relevant adverse event affecting $40 \%$ of fostamatinib-treated subjects with other common negative responses, including, diarrhea, neutropenia and increased hepatic enzyme levels. Some of the fostamatinib-treated RA subjects also developed infections. Most critically, although RA subjects treated with fostamatinib showed a positive clinical response by ACR criteria, none of the 3 clinical trials revealed any significant effects on erosive bone damage over the 6 months of treatment.

Although SyK was shown to play an influential role in regulating the aberrant proliferation of several immune cell types critical to the RA process in vitro and ex vivo, the results of several RA clinical trials with fostamatinib were not impressive enough to warrant further development of this SyK SMI for RA. 


\section{A perspective on the future development of protein kinase SMIs for RA}

The current state of affairs regarding the future development of additional protein kinase small molecule compounds for the treatment of RA should arise from the paradigm employed that ultimately led to the US FDA approving tofacitinib for moderate-to-severe RA. An assessment of the extent to which tofacitinib would be regularly prescribed for the treatment of RA recently concluded that most-marketing surveillance data will ultimately determine the extent to which tofacitinib will only be used to treat RA patients with inadequate responses to conventional DMARDs and/or the several types of biologic drugs now available to treat RA or whether tofacitinib will be employed as a first-line therapy for RA [50]. Although this assertion appears to have some validity based on the current thinking by rheumatologists regarding the use of tofacitinib, decisions must be made by the biopharmaceutical industry as to whether to develop other JAK SMIs for future therapy of autoimmune diseases. Some of these newer small molecule compounds were shown to inhibit JAK1 and JAK3, whereas others may be designed to selectively inhibit JAK2. In that respect, the structure of the JAK3 enzyme may be instructive (Figure 4). It was recently pointed out that tofacitinib was originally introduced as a JAK3selective SMI, but in reality tofacitinib also inhibits JAK1 and JAK2 [100]. In addition, Chrencik et al. [101] indicated that computational analysis comparing tofacitinib with the TyK2 SMI, CMP-6, showed that kinome-selectivity will be a challenge as a consequence of the overall similarities in structure between JAKs 1-3 and TyK2, as well as the fact that the JAK3 SMI binds to the ATP-binding cavities in an orientation which is similar to JAK1 and JAK2. Therefore, the jury is still out, so to speak, as to whether or not immune-mediated inflammation and other pathophysiologic abnormalities associated with aberrant JAK/STAT signaling in RA can be regulated by focusing on developing a JAK SMI specific for a single member of the JAK family or whether small molecule compounds which are designed to inhibit more than one JAK form would be a more effective RA therapy.

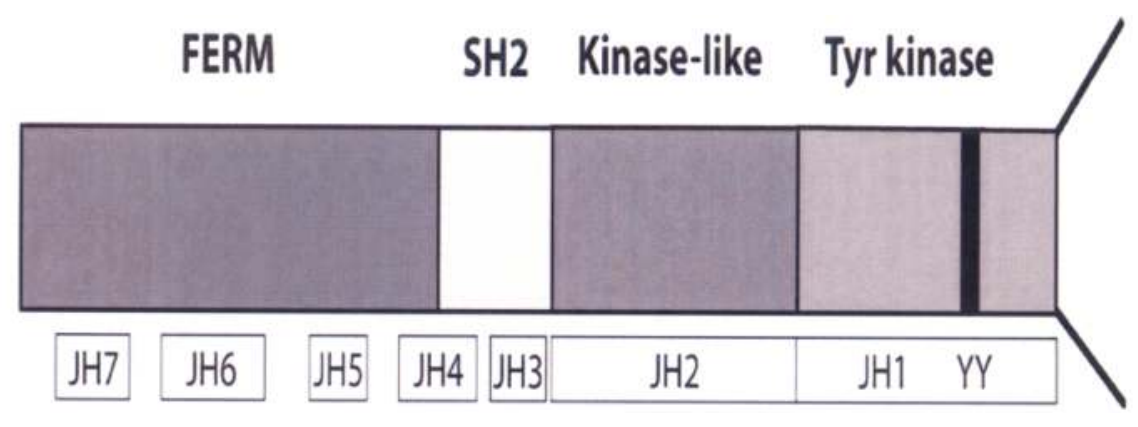

Figure 4. The JH domains and phosphorylation sites of JAK3 [45]

The drug armamentarium for treating RA now include, immunosupressants such as methotrexate, as well as immunomodulatory drugs which block TNF- $\alpha$, anti-IL-1, and T-and B-cell activation among other pathways. Therefore the extent to which JAK and/or SyK inhibitors 
will be employed with equal footing to these well-established drugs with clinical efficacy for RA remains to be determined.

Then there is a host of data that supports a role for other protein kinases in the RA process. However, it can be stated at this time that the results of preclinical and RA clinical trials analyses are more compelling for developing small molecule compounds to inhibit TyK2 than SyK.

Recently, we pointed out that in addition to JAK, TyK2 and SyK, it may be prudent for investigators to consider the role that abnormalities play in other signal transduction pathways associated with RA as future targets for therapeutic intervention [40]. The data supporting a robust level of "cross-talk" between several signaling pathways that contribute to cytokine, matrix metalloproteinase, pro-apoptosis and anti-apoptosis gene expression is well-proven $[28,29,35,40,41]$ and should be taken into account. In that regard, aberrations found in RA in the PI3K/Akt/mTOR pathway, transforming growth factor kinase-1, bone marrow kinase, nuclear factor $\kappa \mathrm{B}$-inducing kinase and Bruton's tyrosine kinase could become a focus of future small molecule inhibitor drug development.

\section{Author details}

Charles J. Malemud*

Address all correspondence to: cjm4@cwru.edu

Department of Medicine, Division of Rheumatic Diseases, Case Western Reserve University, School of Medicine, Cleveland, Ohio, USA

\section{References}

[1] Feely MG, Erickson A, O'Dell JR. Therapeutic options for rheumatoid arthritis. Expert Opin Pharmacother 2009 10(13) 2095-2106.

[2] Upchurch KS, Kay J. Evolution of treatment for rheumatoid arthritis. Rheumatology (Oxford) 2012; 51 Suppl 6 vi28-vi36.

[3] Taherian E, Rao A, Malemud CJ, Askari A. The biological and clinical activity of antimalarial drugs in autoimmune disorders. Curr Rheum Rev 2013 9(1) 45-62.

[4] Braun J. Methotrexate: optimizing the efficacy in rheumatoid arthritis. Ther Adv Musculoskelet Dis 2011; 3(3) 151-158.

[5] Kumar P, Banik S. Pharmacotherapy options in rheumatoid arthritis. Clin Med Insights Arthritis Musculoskelet Disord 2013; 6 35-43. 
[6] Finckh A, Dehler S, Gabay C. The effectiveness of leflunomide as a co-therapy of tumor necrosis factor inhibitors in rheumatoid arthritis; a population-based study. Ann Rheum Dis 2009; 68(1) 33-39.

[7] Meier FM, Frerix M, Hermann W, Müller-Ladner U. Current immunotherapy in rheumatoid arthritis. Immunotherapy 2013; 5(9): 955-974.

[8] Gibbons LJ, Hyrich KL. Biologic therapy for rheumatoid arthritis; clinical efficacy and predictors of response. Biodrugs 2009; 23(2) 111-124.

[9] Östör AJ. Abatacept: a T-cell co-stimulation modulator for the treatment of rheumatoid arthritis. Clin Rheumatol 2008; 27 (11) 1343-1353.

[10] Astry B, Harberts E, Moudgil KD. A cytokine-centric view of the pathogenesis and treatment of autoimmune arthritis. J Interferon Cytokine Res 2011; 31(12) 927-940.

[11] Mertens M, Singh JA. Anakinra for rheumatoid arthritis: a systematic review. J Rheumatol 2009; 36(6) 1118-1125.

[12] Tanaka T, Kishimoto T. Immunotherapy of tocilizumab for rheumatoid arthritis. J Clin Cell Immunol 2013; S6 001

[13] Malemud CJ. Targeted drug development for rheumatoid arthritis. Future Med Chem 2012; 4(6) 701-703

[14] Taylor PC. Pharmacology of TNF blockade in rheumatoid arthritis and other chronic inflammatory diseases. Curr Opin Pharmacol 2010; 10(3) 308-315.

[15] Geyer M, Müller-Ladner U. Actual status of antiinterleukin-1 therapies in rheumatic diseases. Curr Opin Rheumatol 2010; 22(3) 246-251.

[16] Giatromanolaki A, Sivridis E, Athanassou N et al. The angiogenic pathway 'vascular endothelial growth factor/flk-1(KDR)-receptor' in rheumatoid arthritis. J Pathol 2001; 194(1) 101-108.

[17] Malemud CJ, Reddy SK. Targeting cytokines, chemokines and adhesion molecules in rheumatoid arthritis. Curr Rheum Rev 2008; 4 219-234.

[18] Agawal SK, Brenner MB. Role of adhesion molecules in human synovial inflammation. Curr Opin Rheumatol 2006; 18(3) 268-276.

[19] Szekanecz Z, Koch AE. Vascular endothelium and immune responses: implications for inflammation and angiogenesis. Rheum Dis Clin North Am 2004; 30(1) 97-114.

[20] Lee DM, Kiener HP, Agarwal SK et al. Cadherin-11 in synovial lining formation and pathology in arthritis. Science 2007; 315(5814) 1006-1010.

[21] Haringman JJ, Kraan MC, Smeets TJM, Zwinderman KH, Tak PP. Chemokine blockade and chronic inflammatory disease: proof of concept in patients with rheumatoid arthritis. Ann Rheum Dis 2003; 62(8) 715-721. 
[22] Haringman JJ, Gerlag DM, Smeets TJM et al. A randomized controlled trial with antiCCL2 (anti-monocyte chemotactic protein-1) monoclonal antibody in patients with rheumatoid arthritis. Arthritis Rheum 2006; 54(8) 2387-2392.

[23] Liu J, Merritt JR. CC chemokine receptor small molecule antagonists in the treatment of rheumatoid arthritis and other diseases: a current view. Curr Top Med Chem 201; 10 (13) 1250-1267.

[24] White GE, Iqbal AJ, Greaves DR. CC chemokine receptors and chronic inflammation -therapeutic opportunities and pharmacological challenges. Pharmacol Rev 2013; 65(1) 47-89.

[25] Ellingsen T, Hansen I, Thorsen J et al. Upregulated baseline plasma CCL19 and CCR7 cell-surface expression on monocytes in early rheumatoid arthritis normalized during treatment and CCL19 correlated with radiographic progression. Scand J Rheumatol 2014; 43(2) 91-100

[26] Antonelli A, Ferrari SM, Giuggioli D, Ferrannini E, Ferri C, Fallahi P. Chemokine (CX-C motif) ligand (CXCL) 10 in autoimmune diseases. Autoimmun Rev 2014; 13(3) 272-280.

[27] Debnath B, Xu S, Grande S, Garofalo A, Neamati N. Small molecule inhibitors of CXCR4. Theranostics 2013; 3(1) 47-75.

[28] Wylie MA, Malemud CJ. Perspective: Deregulation of apoptosis in arthritis by altered signal transduction. Int J Clin Rheumatol 2013; 8(4) 483-490.

[29] Malemud CJ. Intracellular signaling pathways in rheumatoid arthritis. J Clin Cell Immunol 2013; 4160.

[30] Korb A, Pavenstädt H, Pap T. Cell death in rheumatoid arthritis. Apoptosis 2009; 14(4) 447-454.

[31] Malemud CJ, Sun Y, Pearlman E, Ginley NM, Awadallah A, Wisler BA et al. Monosodium urate and tumor necrosis factor- $\alpha$ increase apoptosis in human chondrocyte cultures. Rheumatology (Sunnyvale) 2012; 2113.

[32] Chang L, Karin M. Mammalian MAP kinase signaling cascades. Nature 2001; 410(6824) 37-40.

[33] Burrage PS, Mix KS, Brinckerhoff CE. Matrix metalloproteinases : role in arthritis. Front Biosci 2006; 11 529-543.

[34] Wu T, Mohan C. The AKT axis as a therapeutic target in autoimmune diseases. Endocr Metab Immune Disord Drug Targets 2009; 9(2) 145-150.

[35] Malemud CJ. The discovery of novel experimental therapies for inflammatory arthritis. Mediators Inflamm 2009; 698769. 
[36] Malemud CJ. Regulation of chondrocyte matrix metalloproteinase gene expression. 2014; In: Dhalia NS, Chakraborti S (Editors) Role of Proteases in Health and Disease. Springer Science, UK.

[37] Malemud CJ. Small molecular weight inhibitors of stress-activated and mitogen-activated protein kinases. Mini Rev Med Chem 2006; 6(6) 689-698.

[38] Malemud CJ: Inhibitors of stress-activated/mitogen-activated protein kinase pathways. Curr Opin Pharmacol 2007; 7(3) 339-343.

[39] Malemud CJ. Defining novel targets for intervention in rheumatoid arthritis: An overview. Curr Rheum Rev 2008; 4 214-218.

[40] Malemud CJ. Dysfunctional immune-mediated inflammation in rheumatoid arthritis dictates that development of anti-rheumatic disease drugs target multiple intracellular signaling pathways. Anti-Inflammatory Anti-Allergy Agents Med Chem 2011; 10(2) 78-84.

[41] Wisler BA, Dennis JE, Malemud CJ. New organ-specific pharmacological strategies interfering with signaling pathways in inflammatory disorders/autoimmune disorders. Curr Signal Transduct Ther 2011; 6 279-291.

[42] Yokota A, Narazaki N, Shima Y et al. Preferential and persistent activation of the STAT1 pathway in rheumatoid synovial fluid cells. J Rheumatol 2001; 28(9) 1952-1959.

[43] Kasperkovitz PV, Verbeet NL, Smeets TJ et al. Activation of the STAT1 pathway in rheumatoid arthritis. Ann Rheum Dis 2004; 63(3) 233-239.

[44] Walker JG, Ahern MG, Coleman M et al. Characterisation of a dendritic cell subset in synovial tissue which strongly expresses Jak/STAT transcription factors from patients with rheumatoid arthritis. Ann Rheum Dis 2007; 66(8) 992-999.

[45] Malemud CJ, Pearlman E. Targeting JAK/STAT signaling pathway in inflammatory diseases. Curr Signal Transduct Ther 2009; 4 201-221.

[46] Malemud CJ. Differential activation of JAK enzymes in rheumatoid arthritis and autoimmune disorders by proinflammatory cytokines - potential drug targets. Int J Interferon Cytokine Mediator Res 2010; 2 97-111.

[47] Malemud CJ. Suppression of pro-inflammatory cytokines via targeting of STAT-responsive genes. 2013; In: El-Shemy H (Editor), Drug Discovery. InTech, Croatia.

[48] Laurence A, Pesu M, Silvennoinen O, O'Shea J. JAK kinases in health and disease. An update. Open Rheumatol J 2012; 6 232-244.

[49] Malemud CJ. Inhibitors of JAK for the treatment of rheumatoid arthritis: Rationale and clinical data. Clin Invest 2012; 239-47. 
[50] Malemud CJ, Blumenthal DE: Protein kinase small molecule inhibitors for rheumatoid arthritis: Medicinal chemistry/Clinical perspectives. World J Orthopedics: 2014; 5(4) 496-503.

[51] Cutulo M, Meroni M. Clinical utility of the oral JAK inhibitor tofacitinib in the treatment of rheumatoid arthritis. J Inflamm Res 2013; 6: 129-136.

[52] Thoma G, Nuninger F, Falchetto R, Hermes E, Tavares GA, Vangrevelinghe E et al. Identification of a potent Janus kinase 3 inhibitor with high selectivity within the Janus kinase family. J Med Chem 2011; 54(1) 284-288.

[53] Smolen JS, van der Heijde D, Machold KP, Aletaha D, Landewé R. Proposal for a new nomenclature of disease-modifying antirheumatic drugs. Ann Rheum Dis 2014; 73(1) 3-5.

[54] Vafadari R, Weimar W, Baan CC. Phosphospecific flow cytometry for pharmacodynamic drug monitoring: analysis iof the JAK-STAT signaling pathway. Clin Chim Acta 2012; 413(17-18) 1398-1405.

[55] Stump KL, Lu LD, Dobrzanski P, Serdikoff C, Gingrich DE, Dugan BJ et al. A highly selective, orally active inhibitor of Janus kinase 2, CEP-33779, ablates disease in 2 mouse models of rheumatoid arthritis. Arthritis Res Ther 2011; 13(2) R68.

[56] Fridman JS, Scherle PA, Collins R, Burn TC, Li Y, Covington MB et al. Selective inhibition of JAK1 and JAK2 is efficacious in rodent models of arthritis: preclinical characterization of INCB028050. J Immunol 2010; 184(9) 5298-5307.

[57] Migita K, Izumi Y, Torigoshi T, Satomura K, Izumi M, Nishino Y et al. Inhibition of Janus kinase/signal transducer and activator of transcription (JAK/STAT) signalling pathway in rheumatoid synovial fibroblasts using small molecule compounds. Clin Exp Immunol 2013 174(3) 356-363.

[58] Norman P. Selective JAK inhibitors in development for rheumatoid arthritis. Expert Opin Investig Drugs 2014; 23(8) 1067-1077.

[59] Hsu L, Armstrong AW. JAK inhibitors: treatment efficacy and safety profile in patients with psoriasis. J Immunol Res 2014; 2014283617.

[60] Liu KD, Gaffen SL, Goldsmith MA, Greene WC. Janus kinases in interleukin-2 mediated signaling: JAK1 and JAK3 are differentially regulated by tyrosine phosphorylation. Curr Biol 1997; 7(11) 817-826.

[61] Haan C, Rolvering C, Raulf F, Kapp M, Drückes P, Thomas G et al. Jak1 has a dominant role over Jak3 in signal transduction through $\gamma$ c-containing cytokine receptors. Chem Biol 2011; 18(3) 314-323.

[62] O'Sullivan LA, Liongue C, Lewis RS, Stephenson SE, Ward AC. Cytokine receptor signaling through the Jak-Stat-Socs pathway in disease. Mol Immunol 2007; 44(10) 2497-2506. 
[63] Himpe E, Kooijman R. Insulin-like growth factor-1 receptor signal transduction and the Janus Kinase/Signal Transducer and Activator of Transcription (JAK-STAT) pathway. Biofactors 2009; 35(1) 76-81.

[64] Vaddi K, Luchi M. JAK inhibition for the treatment of rheumatoid arthritis: a new era in oral DMARD therapy. Expert Opin Investig Drugs 2012; 21(7) 961-973.

[65] Dymock BW, See CS. Inhibitors of JAK2 and JAK3: an update on the patent literature 2010-2012. Expert Opin Ther Pat 2013; 23(4) 449-501.

[66] Kaur K, Kalra S, Kaushal S. Systematic review of tofacitinib: a new drug for the management of rheumatoid arthritis. Clin Ther 2014; 36(7) 1074-1086.

[67] Kawalec P, Mikrut A, Wiśniewska N, Pilic A. The effectiveness of tofacitinib: a novel Janus kinase inhibitor, in the treatment of rheumatoid arthritis: a systematic review and meta-analysis. Clin Rheumatol 2013; 32(10) 1415-1424.

[68] Lee FB, Fleischmann R, Hall S, Wilkinson B, Bradley JD, Gruben D et al. Tofacitinib versus methotrexate in rheumatoid arthritis. N Engl J Med 2014; 370(25) 2377-2386.

[69] Meyer DM, Jesson MI, Li X, Elrick MM, Funckes-Shippy CL, Warner JD et al. Antiinflammatory activity and neutrophil reductions mediated by the JAK1/JAK3 inhibitor, CP690,550 in rat adjuvant-induced arthritis. J Inflamm (London) 2010; 741.

[70] Yamaoka K, Tanaka Y. Targeting the Janus kinases in rheumatoid arthritis: focus on tofacitinib. Expert Opin Pharmacother 2014; 15(1) 103-113.

[71] Vyas D, O’Dell KM, Bandy JL, Boyce EG. Tofacitinib: the First Janus kinase (JAK) inhibitor for the treatment of rheumatoid arthritis. Ann Pharmacother 2013; 47(11) 1524-1531.

[72] Gaujoux-Viala C, Nam J, Ramiro S, Landewé B, Buch MH, Smolen JS et al. Efficacy of conventional synthetic disease-modifying antirheumatic drugs, glucocorticoids and tofacitinib: a systematic literature review informing the 2013 update of the EULAR recommendations for management of rheumatoid arthritis. Ann Rheum Dis 2014; 73(3) 510-515.

[73] http://pubchem.ncbi.nlm.nih.gov/summary/summary.cgi?cid=44205240 (Accessed 10/17/14)

[74] van Vollenhoven RF. Small molecular compounds in development for rheumatoid arthritis. Curr Opin Rheumatol 2013; 25(3) 391-397.

[75] Shi JG, Chen X, Lee F, Emm T, Scherle PA, Lo Y et al. The pharmacokinetics, pharmacodynamics and safety of baricitinib, an oral JAK1/2 inhibitor, in healthy volunteers. J Clin Pharmacol 2014 ; 54(12) 1354-1361.

[76] http://newdrugapprovals.org/2014/07/21/decernotinib-jak-inhibitor-for the treatment of autoimmune and inflammatory disease/ Accessed 8/20/14 
[77] https://b-com.mci-group.com/AbstractList/EULAR2014.aspx Lack of differentiation of Janus kinase inhibitors in rheumatoid arthritis based on Janus kinase pharmacology and clinically meaningful concentrations. Accessed 8/20/14

[78] EULAR Meeting-Van Vollenhoven R et al. Ann Rheum Dis 2014; 73 Suppl 2 Accessed 8/20/14

[79] Malemud CJ. Suppression of autoimmune arthritis by small molecule inhibitors of the JAK/STAT pathway. Pharmaceuticals 2010; 3 1446-1455.

[80] Norman P. Selective JAK 1 inhibitor and selective Tyk2 inhibitor patents. Expert Opin Ther Pat 2012; 22(10) 1233-1249.

[81] Malerich JP, Lam JS, Hart B, Fine RM, Klebansky B, Tanga MJ et al. Diamino-1,2,4triazole derivatives are selective inhibitors of TYK2 and JAK1 over JAK2 and JAK3. Bioorg Med Chem Lett 2010; 20(24) 7454-7457.

[82] Dalila AS, Mohd Said MS, Shaharir SS, Asrul AW, Low SF, Shamsul AS et al. Interleukin-23 and its correlation with disease activity, joint damage, and functional disability in rheumatoid arthritis. Kaohsiung J Med Sci 2014; 30(7) 337-342.

[83] Boniface K, Blom B, Liu YJ, de Waal Malefyt R. From interleukin-23 to T-helper 17 cells: human T helper cell differentiation revisited. Immunol Rev 2008; 226 132-146.

[84] Hayakawa N, Noguchi M, Takeshita S, Eviryanti A, Seki Y, Nishio H et al. Structureactivity relationship study, target identification, and pharmacological characterization of a small molecular IL-12/IL-23 inhibitor, APY0201. Bioorg Med Chem 2014; 22(11) 3021-3029.

[85] Tang C, Chen S, Qian H, Huang W. Interleukin-23: as a drug target for autoimmune inflammatory diseases. Immunology 2012; 135(2) 112-124.

[86] Liang Y, Zhu Y, Xia Y, Peng H, Yang XK, Liu YY et al. Therapeutic potential of tyrosine kinase 2 in autoimmunity. Expert Opin Ther Targets 2014; 18(5) 571-580.

[87] Qi Z, Shen L, Zhou H, Jiang Y, Lan L, Luo L et al. Phosphorylation of heat shock protein 27 antagonizes TNF- $\alpha$ induced HeLa cell apoptosis via regulating TAK1 ubiquitination and activation of p38 and ERK signaling. Cell Signal 2014; 26(7) 1616-1625.

[88] Malemud CJ, Haque A, Louis NA, Wang J: Immune response and apoptosis - Introduction. J Clin Cell Immunol 2012; S3: e001.

[89] Malemud CJ. Role of nonreceptor tyrosine and threonine kinase inhibitors. 2009; In. Gracia-Foncillas J. (Editor) Molecular Biology of Cancer: Toward New Therapies. Prous Science, Barcelona.

[90] Wong BR, Grossbard D, Payan G, Masuda ES. Targeting Syk as a treatment for allergic and autoimmune disorders. Expert Opin Investig Drugs 2004; 13(7) 743-762. 
[91] Scott DL. Role of spleen tyrosine kinase inhibitors in the management of rheumatoid arthritis. Drugs 2011; 71(9) 1121-1132.

[92] Gomez-Puerta JA, Mócsai A. Tyrosine kinase inhibitors for the management of rheumatoid arthritis. Curr Top Med Chem 2013; 13(6) 760-773.

[93] Pine RR, Chang B, Schoettler N, Banquerigo ML, Wang S, Lau A et al. Inflammation and bone erosion are suppressed in models of rheumatoid arthritis following treatment with a novel SyK inhibitor. Clin Immunol 2007; 124(3) 244-257.

[94] http://pubchem.ncbi.nlm.nih.gov/summary/summary.cgi?cid=25008120 （accessed $10 / 17 / 14)$

[95] Boström E, Ohrn F, Hanze E, Sandström M, Martin P, Wählby-Hamrén U. Exposure vs. response of blood pressure in patients with rheumatoid arthritis following treatment with fostamatinib. J Clin Pharmacol 2014 ; 54(12) 1337-1346 doi: 10.1002/jcph. 341

[96] Baloum M, Grossbard EB, Mant T, Lau DT. Pharmacokinetics of fostamatinib, a spleen tyrosine kinase (SYK) inhibitor, in healthy human subjects following a single and multiple oral dosing in three phase I subjects. Br J Clin Pharmacol 2013; 76(1) 78-88.

[97] Platt AM, Benson RA, Mc Queenie R, Butcher JP, Braddock M, Brewer JM et al. The active metabolite of spleen tyrosine kinase inhibitor fostamatinib abrogates the CD4+T cell-priming capacity of dendritic cells. Rheumatology (Oxford) 2014 Jul 26 pii.keu273 [Epub ahead of print]

[98] Taylor PC, Genovese MC, Greenwood M, Ho M, Nasonov E, Oemar B et al. OSKIRA-4: a phase IIb randomised, placebo-controlled study of the efficacy and safety of fostamatinib monotherapy. Ann Rheum Dis 2014 Jul 29 pii:annrheumdis-2014205361. doi: 10.1136/annrheumdis-2014-205361 [Epub ahead of print]

[99] Scott IC, Scott DL. Spleen tyrosine kinase inhibitors for rheumatoid arthritis: where are we now? Drugs 2014; 74(4) 415-422.

[100] Thoma G, Drückes P, Zerwes G. Selective inhibitors of the Janus kinase Jak3-Are they effective? Bioorg Med Chem Lett 2014; 24(19) 4617-4621 pii:S9060-894X(14)00890-7. doi: 10.1016/j.bmcl.2014.08.046

[101] Chrencik JE, Patny A, Leung IK, Korniski B, Emmons TL, Hall T et al. Structural and thermodynamic characterization of the TYK2 and JAK3 kinase domains in complex with CP690550 and CMP-6. J Mol Biol 2010; 400(3) 413-433. 
